\title{
Development of System Detecting for Motor Abnormalities and Controlling Speed of Drone Using Temperature Sensor
}

\author{
Keita Kihara ${ }^{\mathrm{a}}$, Huimin $\mathrm{Lu}^{\mathrm{b}}$, Shiyuan Yang ${ }^{\mathrm{a}}$, Seiichi Serikawa ${ }^{\mathrm{a}}$ \\ ${ }^{a}$ Department of Electrical and Electronics Engineering, Kyushu Institute of Technology, Japan \\ ${ }^{b}$ Department of Mechanical and Control Engineering, Kyushu Institute of Technology, Japan \\ *Corresponding Author: airgear7025@gmail.com
}

\begin{abstract}
Aerial unmanned vehicle is widely used in many fields, such as weather observation, framing, inspection of infrastructure, monitoring of disaster areas. However, the current aerial unmanned vehicle is difficult to avoid falling in the case of failure. The purpose of this paper is to develop an anomaly detection system, which prevents the motor from being used under sharp change in motor temperature rise. In the anomaly detection system, temperature information of the motor is obtained by DS18B20 sensors. We also build a user interface to open the screen of Raspberrypi on laptop for observation. In the experiments, experimental results demonstrate that the proposed system is possibility for unmanned flight safely by controlling drone's speed from information obtained by attaching temperature sensors.
\end{abstract}

Keywords: Aerial unmanned vehicle; Anomaly detection; Temperature sensor

\section{Introduction}

Unmanned aerial vehicles flying through the air is unattended and controlled remotely. Drone, one kind of unmanned aerial vehicles, is widely used, such as, weather observation, spraying of agricultural chemicals, inspection of infrastructure, monitoring of disaster areas.

By Nikkei BP Clean Tech Laboratories, from 2017 to 2030, annual sales volume is predicted to increase from 500 units to 3000 units ${ }^{(1)}$. Performance is expected to improve rapidly in the meantime. As an example of specific performance improvement, the flight time of the current drones is 15 minutes, but it is predicted to be able to fly for 2 hours after 10 years. Figure 1 shows the trends in the number of drone sales units by sector for businesses.

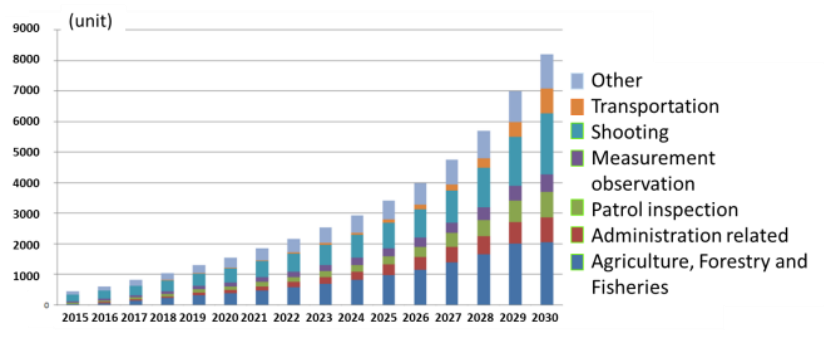

Figure 1 Trends in sales volume of drone

Expansion of the flight time may cause overload on the motor. Overload to the motor leads to motor's failure. From this, we thought that a system to cope with overload is necessary so as not to cause motor's failure. In this paper, we define the case where the temperature rise of the motor is rapidly increasing as an overload condition because the sudden change of motor temperature rise causes the insulation performance deterioration inside the motor, shortening the life and burning the windings.

We propose a motor anomaly detection and speed control system using temperature sensors and ARM Cortex-A53 CPU (Raspberrypi 2B) for the purpose of controlling the usage of abnormal temperature of motor. Temperature sensors are adhered to the motor unit of the drone. The abnormal temperature of the motors is decided on the Raspberrypi, which is set in the drone. A system that adjusts the speed of the drone according to the degree of temperature rise of the motor is implemented for the purpose of countermeasures against sudden change of the motor temperature rise leading to falling. This system prevents the motor temperature from easily rising easily. Also, in order to monitor the output of Raspberrypi 's processing attached to the drone, I connected the terrestrial PC and Raspberrypi remotely.

The organization of this paper in the following is: In section 2 , some related works about motor anomaly detection 
are reviewed. Section 3 shows the system configuration of the proposed system. Experimental results are shown in Section4. Finally, we conclude this paper in Section 5.

\section{Related Works}

There are many methods for detecting anomaly of the motor. In this section, we summarize all of these methods and discuss the benefits and disadvantages of these methods.

Firstly, there is an inspection system by abnormal sound detection (2). It is a method to use the abnormal sound inspection in case-based identification algorithm. In this method, it is necessary to calculate the voice data by feature vector beforehand and calculate it based on the establishment distribution model, which is a kind of supervised learning. Supervised learning requires a training dataset of sample elements along with the desired classification of each of these samples. However, in most cases, it is difficult to train the datasets in extreme environments. So, wrong detection is caused using sound inspection in high noisy background.

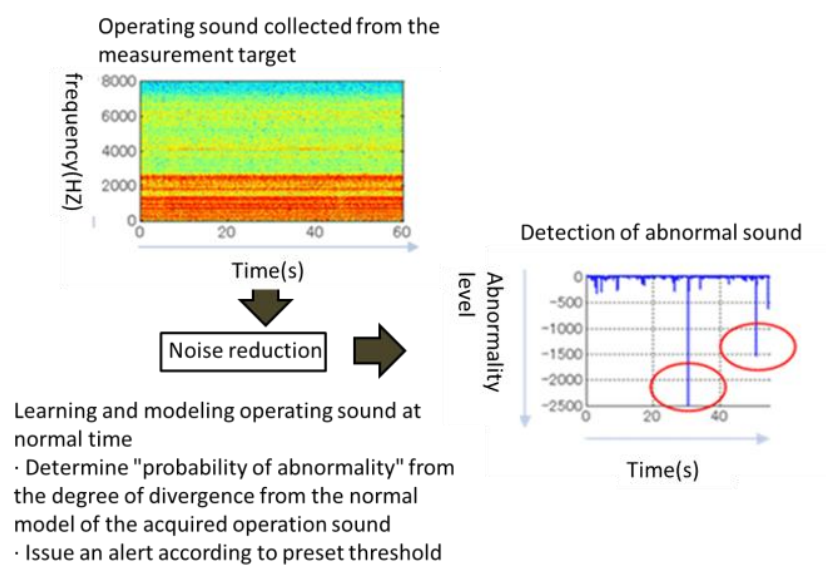

Fig. 2 Inspection system using abnormal sound detection

Secondly, there is a system in which a motor is photographed and an alarm is output when the detected temperature becomes same or higher than the threshold temperature in the infrared image ${ }^{(3,4)}$. This system has a problem that it is difficult to set a thermal imaging camera around the drone. It is difficult to both install a camera to capture a small infrared image and determine the temperature change through low quality infrared images.

As described in above, the conventional anomaly detection methods have the disadvantages in detecting abnormality of the droning motors. In this paper, it was made possible to develop an anomaly detection system on a drone which is difficult with these methods using a temperature sensor (DS18B20), and Raspberrypi 2B.

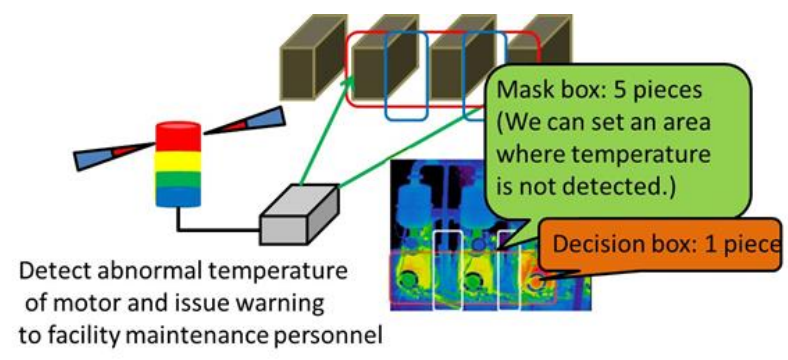

Fig.3 Thermal imaging based abnormal temperature detection system.

\section{System Configuration}

In this study, one abnormal state is defined. When the degree of temperature rise of the motor exceeds the set temperature, it is defined as a rise abnormality, and the temperature at this time is set as the rising threshold value. An object of the present invention is to prevent failure of a motor by preventing a sudden rise in temperature of the motor. For this purpose, we measure the temperature rise per time of the motor and build a system to adjust the speed of the drone when the dynamically changing rising threshold is exceeded.

\subsection{System Overview}

Figure 4 shows overview of system flow. The model proposed in this research consists of two devices, a drone real machine equipped with what is necessary for experiments and a hardware for observation. The drone side is equipped with a temperature sensor and Raspberrypi. Hardware for observation use laptop. A block diagram of the system is shown in Figure 5.

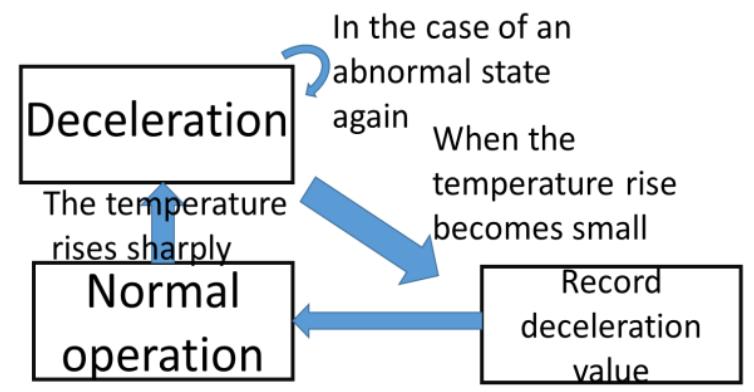

Fig. 4. Overview of system flow 


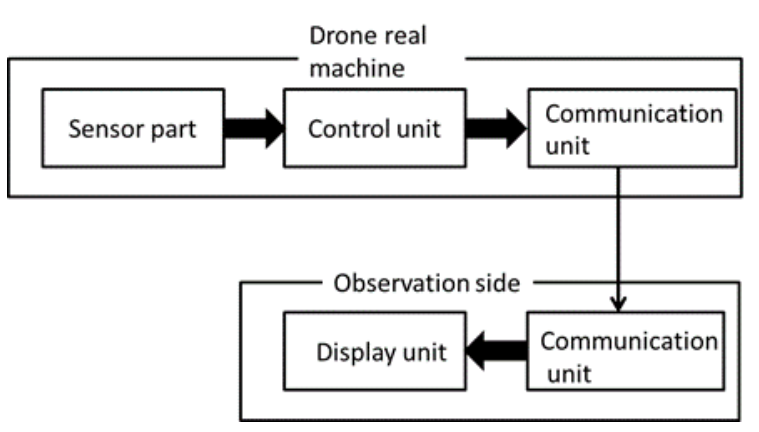

Fig. 5. System working diagram.

The processing sequence of the system is

STEP 1. Make Raspberrypi operable on laptop

STEP 2. Take off instruction from Raspberrypi to drone

STEP 3. After drone takes off, advance

STEP 4. Measure the temperature of the motor part during

flight, also measure the degree of temperature rise every 2 seconds

STEP 5. In the case of normal flying, update the rising threshold for judging the rising abnormality, and decelerate the speed in the case of the rising abnormality

STEP 6. Optimum value candidates for deceleration are output at regular time intervals

STEP 7. After outputting three optimum value candidates, the optimum value of deceleration is set as the value that can improve the temperature rise degree the most among the optimum value candidates.

STEP 8. In case of abnormal rise, decelerate with optimum value of deceleration.

\subsection{Around Drones}

Drone Composition: The structure of the drone is made up of four units: a detection unit, an actual machine (drone), a control unit, and a communication unit. A temperature sensor is used for the detection unit and Raspberrypi is used as the control part ${ }^{(5)}$.

\subsection{Specific flow of the system}

Figure6 and Figure7 shows the flow of a drone control system that detects a sudden increase in temperature rise. We will explain the specific flow of the system that adjusts the speed of drone, detecting the rapid increase of temperature rise.

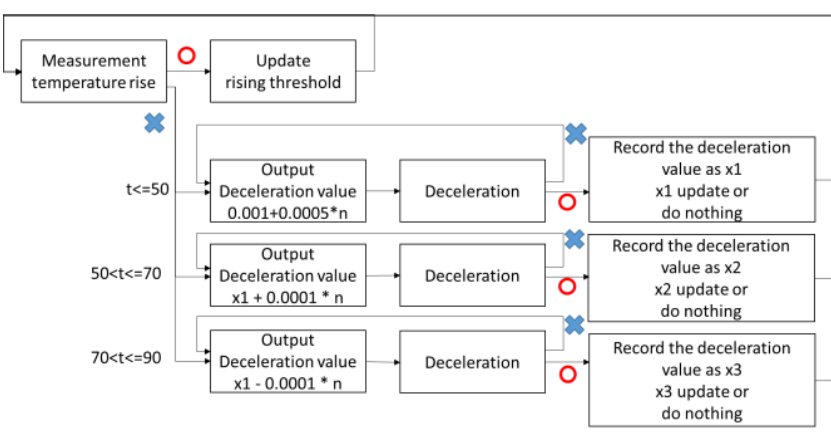

$\mathrm{x}$ 1:Deceleration optimum value candidate $1 \mathrm{t}$ : Time since measurement started x2:Deceleration optimum value candidate 20 Temperature increase degree $\leqq$ Rising threshold value x3:Deceleration optimum value candidate 3 Temperature rise degree> Rise threshold $\mathrm{n}:$ Number of rise abnormality occurrences (every set time)

Fig. 6. Overview of system flow (1)

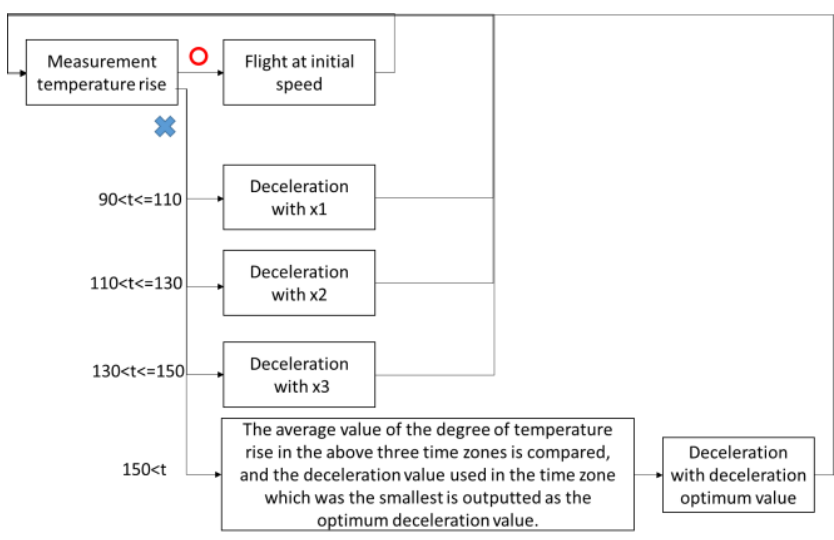

Fig. 7. Overview of system flow (2)

In this system, calculate the degree of temperature rise at 2-second intervals of the drone motor. If the temperature rise does not exceed the rise threshold, the rise threshold is updated. In the case of exceeding, we aim to change the deceleration value depending on the time, and obtain the deceleration value that can improve the rise abnormality among them. The sum of the average value of the degree of temperature rise and the standard deviation is set as the rising threshold. Accordingly, when updating the rising threshold value, the average value and the standard deviation are recalculated including the measurement result, and the rising threshold value is updated. The deceleration method at the time of abnormality, which varies with time, is shown below.

First, decelerate by $0.001+0.0005 * \mathrm{n}$ until 50 seconds after the start of measurement. Among them, the deceleration value that improved the rise abnormality is recorded as the optimum deceleration value candidate 1 (hereinafter referred to as $\mathrm{x} 1$ ), and when a deceleration value that can be improved more than the recorded deceleration value appears, $\mathrm{x} 1$ is updated.

Similarly, from 50 seconds to 70 seconds, decelerate by 
$\mathrm{x} 1+0.0001 * \mathrm{n}$. Among them, the deceleration value that improved the rise abnormality is recorded as the optimum deceleration value candidate 2 (hereinafter referred to as $\mathrm{x} 2$ ), and when a deceleration value that can be improved more than the recorded $\mathrm{x} 2$ appears, $\mathrm{x} 2$ is updated.

Even at 70 to 90 seconds, it decelerates x1-0.0001*n. Among them, the deceleration value that improved the rise abnormality is recorded as the optimum deceleration value candidate 3 (hereinafter referred to as $\mathrm{x} 3$ ), and when a deceleration value that can be improved more than the recorded x 3 appears, $\mathrm{x} 3$ is updated.

From 90 seconds to 110 seconds, deceleration is performed at $\mathrm{x} 1$ in the case of a rise abnormality. From 110 seconds to 130 seconds, deceleration is performed with $\mathrm{x} 2$ in the case of a rise abnormality. From 130 seconds to 150 seconds, deceleration is performed at $\mathrm{x} 3$ in the case of rise abnormality. The average value of the degree of temperature rise in case of coping with the same time rise abnormality in each of $x 1, x 2$, and $x 3$ is outputted. Then, the deceleration value used during the time period when the average value was the smallest is outputted as the optimum deceleration value.

In this research, the system was constructed to achieve the purpose of speed adjustment when the temperature rise of the motor was measured every 2 seconds and it was over the rise threshold. Therefore, experiments were conducted to confirm whether this objective was achieved.

The flow of the experiment is as follows.

1. Calculate the optimum deceleration value by speed adjustment of the drones based on the temperature rise of the motor

2. Comparison of the degree of temperature rise in the case of coping with using the optimum deceleration value in the case of rise abnormality and the case of flying at a constant speed

\section{Experiment Result}

1.The results of experiments for determining the optimum value as the deceleration value in the case where the motor temperature rise rate suddenly increases occur is shown.

From 0 seconds to 50 seconds after the start of measurement of the temperature rise degree, it was possible to observe the speed of the drone and the value of the motor temperature rise as shown in Table 1 . The speed of the drones is indicated in the range of 0 to 1 on the program. It counts every time the temperature is measured. Therefore, from 0 seconds to 50 seconds it means from count 0 to count 25 . The degree of temperature rise in the table means the degree of temperature rise from the previous count.

Table 1. Measurement results on count 1 to 25

\begin{tabular}{|c|c|c|}
\hline count & speed & Temperature rise degree $\left({ }^{\circ} \mathrm{C}\right)$ \\
\hline 1 & 0.05 & -0.125 \\
\hline 2 & 0.05 & -0.125 \\
\hline 3 & 0.05 & 0.063 \\
\hline 4 & 0.05 & 0.062 \\
\hline 5 & 0.05 & -0.125 \\
\hline 6 & 0.05 & 0.063 \\
\hline 7 & 0.0485 & 0.125 \\
\hline 8 & 0.048 & 0.062 \\
\hline 9 & 0.0475 & 0.125 \\
\hline 10 & 0.047 & 0.125 \\
\hline 11 & 0.0465 & 0.125 \\
\hline 12 & 0.046 & 0 \\
\hline 13 & 0.05 & 0.125 \\
\hline 14 & 0.0455 & 0 \\
\hline 15 & 0.05 & 0.188 \\
\hline 16 & 0.045 & 0.125 \\
\hline 17 & 0.0445 & 0.188 \\
\hline 18 & 0.044 & 0.188 \\
\hline 19 & 0.0435 & 0 \\
\hline 20 & 0.05 & 0.125 \\
\hline 21 & 0.043 & 0.125 \\
\hline 22 & 0.0425 & 0 \\
\hline 23 & 0.05 & 0.062 \\
\hline 24 & 0.042 & -0.188 \\
\hline 25 & 0.05 & 0.25 \\
\hline
\end{tabular}

From this result, it is possible to set the temperature rise degree after the rise abnormality to the minimum value of 0.188 as the deceleration value to decelerate at the rise abnormality 0.008 is the optimum deceleration at the rise abnormality in the range from count 1 to 25 . It can be judged as a value. Optimum value candidates were also output in each of the subsequent time zones. Then, 0.0084 and 0.0079 were output. In this measurement, 0.008 was output as the optimum deceleration value at the time of rise abnormality.

2. In the case of a constant speed, the temperature rose from $19.375^{\circ} \mathrm{C}$ to $27.687^{\circ} \mathrm{C}$ and was output when raising $25.312^{\circ} \mathrm{C}$ from $18.75^{\circ} \mathrm{C}$ using the system of this study. Therefore, demonstrate that it is possible to suppress the temperature rise compared with the case where the system is not mounted when dealing with the rising abnormality using the optimum value of decelerating at the rise abnormality obtained in Experiment 1 we made it.

\section{Conclusion}

In this paper, we developed a system which detects the sudden temperature rise of the motor and solves the purpose of dealing with deceleration. As a result of actual demonstration of system development of the drone, we output the optimum value as the deceleration value to 
suppress temperature rise when the temperature rapidly rises. It was confirmed that temperature rise can be suppressed at the time of mounting than when not mounted.

\section{References}

(1) Nikkei BP Clean Tech Laboratory: "100 billion yen in 30 years Drone for marketing business, scenario of diffusion", Atsuo Kikuchi, 2015,

http://www.nikkei.com/article/DGXMZO88951410w5 A700C $1000000 / ? \mathrm{df}=2$

(2) NTT DATA "Started providing IOT solution to detect abnormality from working sound" NTT DATA, 2016/10 /3, https://latte.la/column/43321221

(3) Nippon Avionics Co., Ltd,"K-1. Motor abnormal temperature detection system |Infrared thermography |Nippon Avionics" Nippon Avionics Co., Ltd,1999, http://www.avio.co.jp/products/infrared/solution/kheavy/k-01.html

(4) Oriental Motor Co Ltd, "Design life of motor |Technical data |". Oriental Motor Co Ltd, https://www.orientalmotor.co.jp/tech/reference/life01/

(5) Keita Kihara "Development of the system detecting for motor abnormalities of drone using temperature sensor"ICIAE2017,2017,

https://www2.ia-

engineers.org/conference/index.php/iciae/iciae2017/pa per/viewFile/1348/906 